
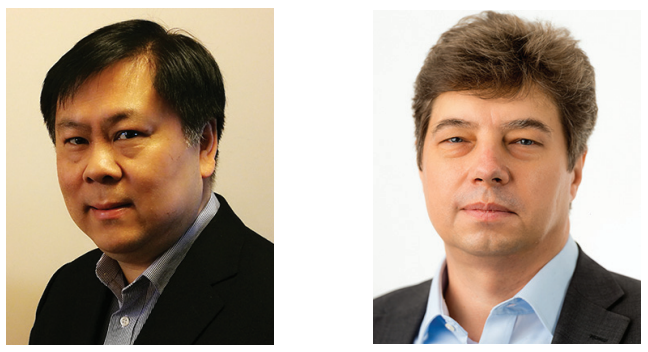

\title{
Advanced Photonics: one year on
}

Advanced Photonics enters its second year. Looking back at our first volume, we feel proud. We have published 29 research articles including 5 reviews, covering all major topics of optical sciences, from imaging and laser physics to quantum optics, metamaterials, and nanophotonics and cutting across both fundamental and applied research. Our editorial board had very difficult decisions to make in rejecting many strong articles, including invited ones, which were still not up to the standards we are aiming at. We hope our authors understand our transparent approach and will continue to support the journal by submitting their best work to us. We have very fast turn-around with on average just 3.2 weeks from the submission to the first decision. The published articles were downloaded more than 100,000 times (!), an astonishing number. While we are awaiting for the journal impact factor, we can report that according to Google Scholar, the articles from Volume 1 were cited on average around 5.2 times during the year of publication. We leave it to the readers to make a comparison with the immediacy factors of other journals. This achievement would not be possible without the trust of our authors and readers and the dedication of the editorial board. Thank you.
Optics and photonics in 2019 were not static and neither were we. With the significant growth in research on quantum optics and quantum optical technologies around the world, we appointed two new editorial board members to better cover this expanding topic of the submissions. Additional editors were also appointed to cover the growing area of applications of two-dimensional materials in photonics.

The journal editors will be present at many conferences throughout the year and we encourage authors and readers to talk to them and learn about new developments with the journal. Or just drop us an email. We are always eager to hear about your new ideas and manuscripts and happy to discuss them with you in advance of submission. We look forward to identifying new opportunities for our authors, the journal, and the optics and photonics community.

Xiao-Cong (Larry) Yuan Co-Editor-in-Chief

Anatoly Zayats Co-Editor-in-Chief

(C) The Authors. Published by SPIE and CLP under a Creative Commons Attribution 4.0 Unported License. Distribution or reproduction of this work in whole or in part requires full attribution of the original publication, including its DOI. [DOI: 10.1117/1 .AP.2.1.010101] 